\title{
Congestion Control Strategies on Integrated Routing Protocol for the Opportunistic Network: A Comparative Study and Performance Analysis
}

\author{
B. Yahaya \\ Department of Electrical and \\ Computer Engineering, \\ Ahmadu Bello University
}

\author{
M.B Mu'azu \\ Department of Electrical and \\ Computer Engineering, \\ Ahmadu Bello University
}

\author{
S. Garba \\ Department of Electrical and \\ Computer Engineering, \\ Ahmadu Bello University
}

\begin{abstract}
This work presents the incorporating of congestion control on the integrated routing protocol of the opportunistic networks. Pre-emptive congestion control strategies were incorporated into the integrated routing protocol. Results showed that the duplication avoidance improved the integrated routing protocol because it reduced the packet loss and improved the delivery probability. Duplication avoidance reduced the packet loss by $58 \%$ and improved the delivery probability by $4 \%$ at the end of the simulation time when compared with the delivery probability and packet loss of the integrated routing protocol without congestion control. The use of acknowledgement, buffer size advertisement, data centric method reduced the packet loss by $2.5 \%, 57 \%$ and $57 \%$ respectively but did not improve on the delivery probability significantly
\end{abstract}

\section{General Terms}

Opportunistic network, congestion control, ONE simulator

\section{Keywords}

Prophet routing protocol, epidemic routing protocol and integrated routing protocol.

\section{INTRODUCTION}

The opportunistic network is an autonomous connection of users that communicate over a relatively bandwidth constrained wireless network [1]. It is a delay tolerant network (DTN) and it consists of nodes which are electronic devices that are attached to a network and are capable of sending, receiving or forwarding information over communication channel [2]. The basic features of the opportunistic networks are: It has wirelessly connected nodes which can be fixed or mobile, A complete path between two nodes aspiring to communicate does not exist, It does not have a fixed communication range, Its routes are dynamically built as any node can opportunistically be used as next hop provided it is likely to bring the message closer to the final destination, Network topology is also flexible as it can change at any time amongst others [3], [4], [5]. The two main routing techniques for opportunistic networks are the context-aware (an example is the Prophet routing protocol) and the context-oblivious (an example is the Epidemic routing protocol). Prophet' is able to learn automatically from the past communications opportunities determined by user's mobility pattern and use context information in the future to route messages. This selflearning feature is absent in 'Epidemic' as it floods the message all over the network, thereby causing congestion and creating high overhead. Epidemic is the only solution when context information about the users is not available

Epidemic is better (in terms of message delay and delivery probability) in absence of context information while 'prophet' gives better result with context information available [6], [7]. It is not certain that a node with a higher metric will be encountered within reasonable time. It may be possible that a destination is new and context information about that destination is not spread in network. To address these problems, the integrated routing approach uses context data as soon as it is available and falls back to dissemination-based routing when context information is not available. It distributes copies of messages to all its neighbors (same as flooding-based techniques), after a configurable time, when nodes have not received any context information about the destination of message. However, it did not consider congestion control and resource utilization which would have improved its performance. [5].

The authors in [8] surveyed the processes of delay tolerant networks congestion control and organised a taxonomy that helped in mapping and comparing the existing DTN congestion control mechanism. They deduced that "there is no universal congestion control mechanism that will be applicable to all DTN scenarios and applications". In the work of [9], integrated buffer management strategy (CIM) was proposed and evaluated using ONE simulator with a view to reducing congestion. But lower delivery ratio and higher overhead was recorded as compared to the spray-and-wait model. Authors in [10] presented a model to help designers carry out fair comparisons among protocols. But the model was not robust because it did not consider more routing protocols. [11] Presented a buffer management strategy to avoid head-of-line blocking in the first-in-first-out case and showed that the proposed strategy reduced the degradation of average delivery delay performance [11].

The opportunistic network environment (ONE) simulator was developed by [12]. It is a customizable java-based simulator targeted for research in DTN routing and application protocol .The rest of the paper is organized as: Section 2 describes congestion in opportunistic network. Section 3 gives the methodology adopted with the simulation setup. Section 4 presents the result obtained and comparison of the congestion control strategies. Section 5 concludes the research.

\section{CONGESTION IN OPPORTUNISTIC NETWORKS}

Opportunistic Network is characterized by sparse connectivity, fault tolerance and forwarding through mobility. Hence, many routing protocols [7], [13], [14], [15], [16] adopt flooding based schemes to deal with the problem of network partitioning and unpredictability in connections in order to increase the message delivery. Node receives packets, stores them in their buffer, carry them when moving and pass them to other nodes when they meet each other. The excessive multi-copies spraying in the network cause serious network congestion by exhausting the nodes, there by influencing the 
performance of transmission [9]. In opportunistic networks, no end-to-end connection is established and therefore congestion cannot be detected and controlled by a feedback loop [17], [18].

With an uncontrolled eviction policy there is a potential risk that all replicated copies of the data may be evicted before all destinations have been reached, hence decreasing the delivery ratio [17]. Congestion occurs when a node's buffer becomes saturated. Lack of end-to end path between the source and the destination coupled with limited resources and high delays effect TCP/IP protocols in opportunistic environments. There is no propagation in the network making many Internet protocols (such as TCP) designed to assume quick return of acknowledgement and data susceptible to failure [19] The challenge is how to avoid congestion without the feedback loop, using just local information at nodes. Avoiding congestion can be done by pre-emptive eviction of data items from the buffers of the nodes. This could be done in the following ways:

\section{i. Buffer eviction using acknowledgement}

The use of acknowledgement in opportunistic network differs from that of legacy network. In the Internet, TCP acknowledgements are used to show that the message has reached its destination hence, the message does not need to be retransmitted and nodes will discard the message from their buffer. But this is not possible in opportunistic networks as there is no end-to-end connectivity. To use acknowledgement in opportunistic network, Time-to-live that is longer than the message must be attached, else, a copy of the message can still live on the network. The advantage with acknowledgement is that nodes are sure that the message has been delivered to the destination before the message is evicted from the buffer. A disadvantage is that it takes time for an acknowledgement to disseminate in the network. Acknowledgements also need time-to-live (TTL) to avoid the packets from lingering in the network [17].

\section{ii. Buffer size advertisement}

Congestion collapse (congestion caused by aggregated undelivered replicated packet that waste bandwidth) can be avoided by nodes sharing their buffer utilization statistics with neighboring nodes. With those statistics, a node can estimate the congestion level at neighboring nodes. By advertising their free buffers, the neighboring nodes can take decision on what to forward and how much to transfer, making it possible to avoid overloading the node and to prioritize message in order to use the buffer space as efficiently as possible. This method of congestion avoidance may increase the delivery time [17].

\section{iii. Data-centric node congestion avoidance}

In the data-centric strategy, messages are forwarded based on the interest in the data. Using this principle in opportunistic networks, it is assumed that a node is more likely to provide buffer space for data items that are of interest to the node itself. It is also assumed that forwarding nodes keep data that they are interested in, which makes the interested forwarding node to become the new source [17]. Nodes choose to evict data that is of little interest to the nodes in the network, as few nodes will request for that data. Nodes can also decide to evict data that is of high interest by assuming that other nodes probably have the data, since it will be frequently requested for and shared. The disadvantage of this strategy is that there is an increase in storage of data items that will never be forwarded or have already reached all nodes interested in the data [17]. Either they are data items that no node is interested in, or data items that have been replicated many times. These data item become stale and consume buffer space, which could have been used to forward other data item.

\section{iv. Duplication Avoidance}

In this strategy, a node keeps only messages it does not have in its buffer. That is, no duplication of messages. When a node comes into communication range with other nodes, it receives a message and checks if it has the same message in its buffer if it has the message; it then deletes the message in order to avoid duplication. This method avoids unnecessary wastage of buffer space by ensuring that no same copies of messages is kept in the buffer.

\section{METHODOLOGY}

The methodology adopted in this research is as follows:

- Modeling of the opportunistic network using the integrated routing protocol.

- Incorporation of the congestion control strategies into the ONE simulator.

- Simulation using the ONE simulator

\subsection{Modeling the Opportunistic Network using the integrated routing protocol.}

The integrated routing protocol was used to model the opportunistic network where every node maintains a delivery probability metric.

The metric is updated whenever a node meets with other nodes using equations (1) to (3) of [20]. When node $\mathrm{x}$ meets node $\mathrm{y}$, the delivery probability of node $\mathrm{x}$ for $\mathrm{y}$ is updated by:

$$
P_{x y}^{\prime}=P_{x y}+\left(1+P_{x y}\right) P_{0}
$$

Where $P_{0}$ is an initial probability $\left(P_{0}\right.$ ranges between 0 and 1$)$, $P^{\prime}{ }_{x y}$ is the current delivery probability of node $\mathrm{x}$ for $\mathrm{y}$ and $P_{x y}$ is former delivery probability of node $\mathrm{x}$ for $\mathrm{y}$. When node $\mathrm{x}$ does not meet with node $y$ for some predefined time, the delivery probability decreases by:

$$
P^{\prime}{ }_{x y}=\gamma^{k} P_{x y}
$$

Where $\gamma$ is the aging factor $(\gamma<1)$, and $\mathrm{k}$ is the number of time units since the last update. When node $\mathrm{x}$ receives node y's delivery probabilities, node $\mathrm{x}$ may compute the transitive delivery probability through $\mathrm{y}$ to $\mathrm{z}$ by:

$$
P_{X Z}^{\prime}=P_{X Z}+\left(1+P_{X Z}\right) P_{x y} P_{Y Z} \beta
$$

Where $\beta$ is a design parameter for the impact of transitivity. $\beta$ $\in[0,1]$.

The values of $P_{0}, \gamma$ and $\beta$ used for this research are $0.75,0.98$ and 0.25 respectively.

\subsection{Incorporating the Congestion Control Strategies}

The congestion strategies that were coded in java and incorporated into the ONE simulator are:

\section{i. Buffer eviction using acknowledgement}

Nodes were made to delete copies of messages from their buffers once they receive an acknowledgement that the message has reached its destination. A TTL of 300 seconds was assigned to the messages. The use of Acknowledgement 
as the means of congestion control was called into the scenario settings of the ONE using the following Code:

\section{Scenario.name $=$ Baseerah_Acknowledgement}

\section{ii. Buffer size advertisement}

Nodes advertise their buffer size so that a sending does not send what the receiving node cannot carry. The use of buffer size advertisement was called into the scenario setting of the ONE using the following Code:

Scenario.name $=$ Baseerah_Advertisement

iii. Data-centric node congestion avoidance

Nodes receive only messages they are interested in, or messages they think it will have high demand from other nodes. The use of Data-centric node congestion avoidance was called into the scenario setting of the ONE using the following Code:

\section{Scenario.name $=$ Baseerah_DataCentric}

\section{iv. Duplication Avoidance}

Here, before a node receives a message from a sending node it must check its buffer to make sure it does not have the message in order not to duplicate the message. The use of Duplication Avoidance was called into the scenario setting of the ONE using the following Code:

\section{Scenario.name $=$ Baseerah_DuplicationAvoidanc}

\subsection{Simulation using $\mathrm{ONE}$}

A part of the Helsinki downtown area as depicted in Figure 1 was used (4500 X 3400m). Communication between modern mobile phones and similar devices (such as, personal digital aid) was assumed. Devices have up to $20 \mathrm{MB}$ of free RAM for buffering messages. Users travel on foot, in cars or trams. Some paths to the parks, shopping malls and trams route have been added. Simulation was run using 100 nodes. Mobile nodes have different speeds and pause times.

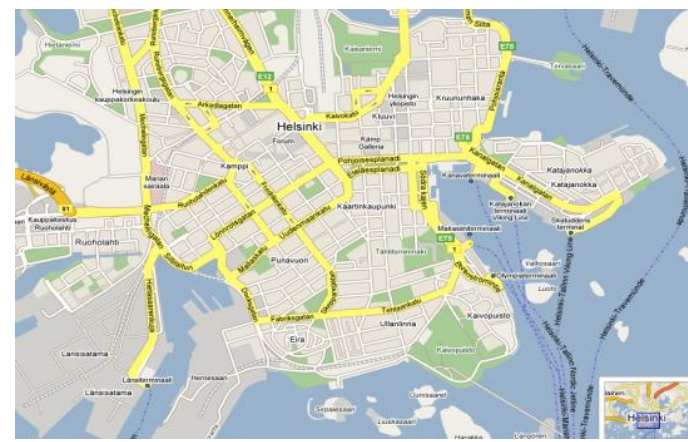

Figure 1: An Overview of the Helsinki Simulation Area

Pedestrians move at random speeds of 0.5 to $1.5 \mathrm{~m} / \mathrm{s}$, taking a pause time between 0 to $120 \mathrm{~s}$. Cars are optional, when present; they make $20 \%$ of node count, moving at a speed of 10 to $50 \mathrm{~km} / \mathrm{h}$, with a pause time of 0 to $120 \mathrm{~s}$. Trams run at a speed of 7 to $10 \mathrm{~m} / \mathrm{s}$, pausing for 10 to 30 s at each configured stop. An assumption of $10 \mathrm{~m}$ range, 2 Mbits Bluetooth and a low power use of $802.11 \mathrm{bWLAN}$ (30m range, $4.5 \mathrm{Mbits}$ ). Mobile users generate messages on an average of once per hour per node. Messages lifetime of 3, 6 and 12 hours were used. The message size used was uniformly distributed between $100 \mathrm{~kb}$ (test message) and 2MB (digital photo). Part or the scenario setting is shown as:
Scenario.name $=$ Baseerah_Integrated

Scenario.simulateConnections $=$ true

Scenario.updateInterval $=1.0$

Scenario.endTime $=43200$

Scenario.nrofHostGroups $=1$

btInterface.type $=$ SimpleBroadcastInterface

btInterface.transmitSpeed $=250 \mathrm{k}$

btInterface.transmitRange $=10$

highspeedInterface.type $=$ SimpleBroadcastInterface

highspeedInterface.transmitSpeed $=10 \mathrm{M}$

highspeedInterface. transmitRange $=1000$

Group. movementModel $=$ RandomWaypoint

Group.router $=$ [ProphetRouter $;$ EpidemicRouter; $]$ \#[Integrated]

ProphetRouter.secondsInTimeUnit $=1$

Group.bufferSize $=10 \mathrm{M}$

Group.nrofInterfaces $=1$

Group.interface $1=$ btInterface

Figure 2 shows the integrated routing simulation carried out on Helsinki map where nodes follow a predefined path and communicates in a transitive manner.

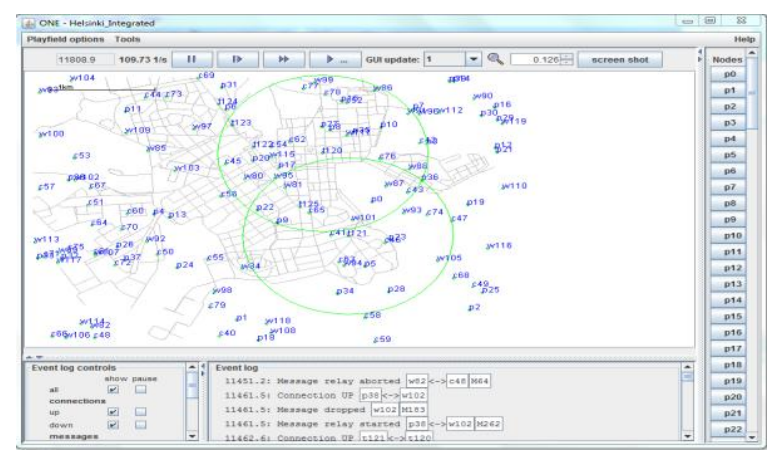

Figure 2: An Overview of the Helsinki Integrated Routing Simulation

\section{RESULT AND DISCUSSIONS}

The results obtained from the application of the four congestion control strategies into the integrated routing protocol on the Helsinki simulation area are shows in Tables 1 and 2, where PL and DP represent packet loss and delivery probability respectively. Table 1 shows the result obtained for the integrated routing protocol without congestion control, while Table 2 shows the result obtained from the different congestion control strategies.

Table1: Integrated Routing Simulation without Congestion Control for Helsinki Region

\begin{tabular}{|c|c|c|}
\hline \multicolumn{3}{|c|}{ Integrated Routing } \\
\hline Sim time (s) & PL & DP \\
\hline 0 & 0 & 0 \\
\hline 1000 & 0 & 0.078 \\
\hline
\end{tabular}




\begin{tabular}{|c|c|c|}
\hline 2000 & 0 & 0.099 \\
\hline 3000 & 6 & 0.082 \\
\hline 4000 & 25 & 0.082 \\
\hline 5000 & 36 & 0.099 \\
\hline 6000 & 48 & 0.102 \\
\hline 8000 & 74 & 0.103 \\
\hline 10000 & 84 & 0.102 \\
\hline 12000 & 119 & 0.101 \\
\hline 14000 & 155 & 0.103 \\
\hline 16000 & 180 & 0.103 \\
\hline 18000 & 192 & 0.103 \\
\hline 20000 & 215 & 0.104 \\
\hline 22000 & 245 & 0.103 \\
\hline 24000 & 280 & 0.104 \\
\hline 26000 & 320 & 0.105 \\
\hline 28000 & 385 & 0.106 \\
\hline 30000 & 420 & 0.106 \\
\hline 32000 & 452 & 0.104 \\
\hline 34000 & 485 & 0.104 \\
\hline 36000 & 519 & 0.103 \\
\hline 38000 & 564 & 0.103 \\
\hline 40000 & 602 & 0.104 \\
\hline 42000 & 648 & 0.1052 \\
\hline 43200 & 670 & 0.1048 \\
\hline
\end{tabular}

It can be deduced from Table 2 that the duplication avoidance performs better with a final PL of 283 and DP of $10.9 \%$ as compared to Table 1 without congestion control strategy having a PL and DP 670 and $10.48 \%$ respectively. The use of acknowledgement, buffer size advertisement and data centric method have PL of 653, 286 and 290 respectively and DP of $0.105,0.103$ and 0.105 respectively. The graphical representation of the improved integrated routing with congestion control as compared with that of the integrated routing protocol without congestion control is shown in
Figures 3 and 4 . Figure 3 shows the variation of packet loss with time while Figure 4 shows the variation of delivery probability with time. Figures 3 and 4 were generated using the Matlab (Matlab 2013Rb) script.

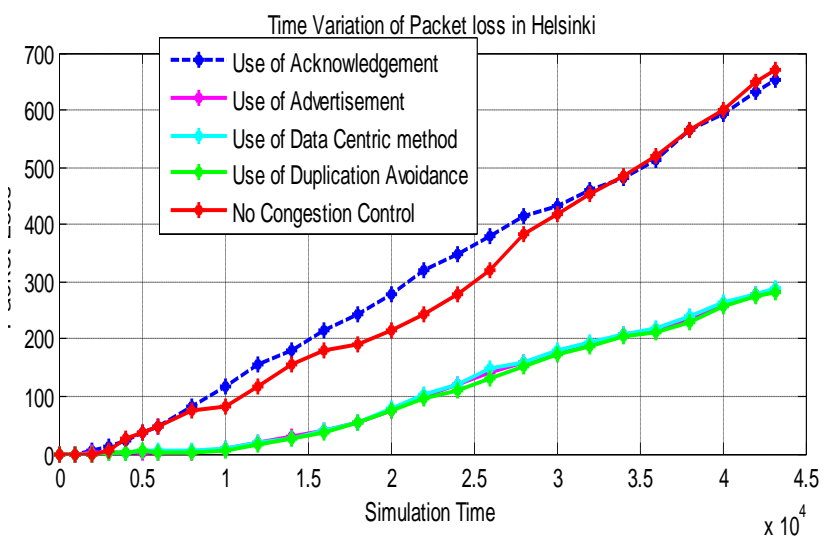

Figure 3: Variation of Packet Loss with Time for Helsinki Region.

Figure 3 shows that buffer size advertisement, data centric method and the avoidance of duplication reduced the packet as compared to the integrated routing protocol without congestion control. The use of acknowledgement reduced the packet loss only towards the end of the simulation time.

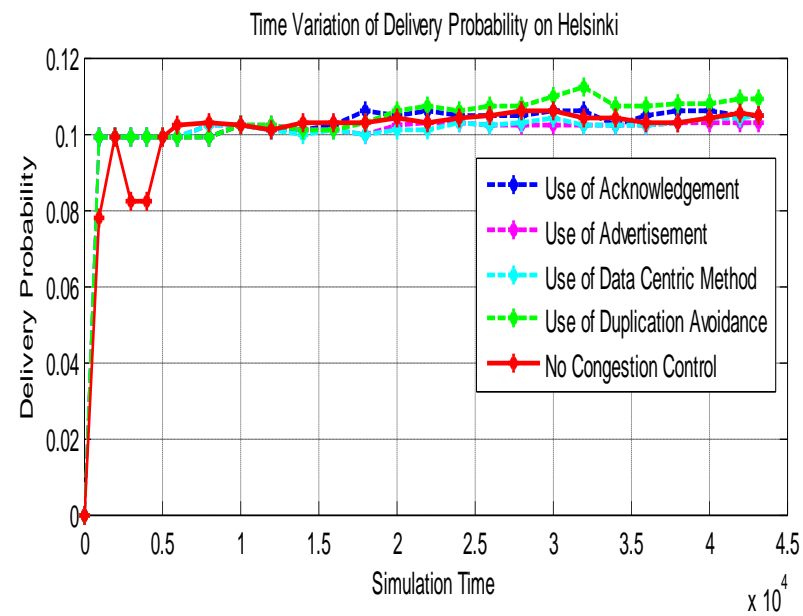

Figure 4: Variation of Delivery Probability with Time for Helsinki Region

Table 2: Improved Integrated Routing With Congestion Control for Helsinki Region

\begin{tabular}{|c|c|c|c|c|c|c|c|c|}
\hline \multirow[b]{2}{*}{$\begin{array}{l}\text { Sim time } \\
\text { (s) }\end{array}$} & \multicolumn{2}{|c|}{ Acknowledgement } & \multicolumn{2}{|c|}{ Advertisement } & \multicolumn{2}{|c|}{ Data Centric } & \multicolumn{2}{|c|}{$\begin{array}{c}\text { Duplication } \\
\text { Avoid }\end{array}$} \\
\hline & PL & DP & PL & DP & PL & DP & PL & DL \\
\hline 0 & 0 & 0 & 0 & 0 & 0 & 0 & 0 & 0 \\
\hline 1000 & 0 & 0.099 & 0 & 0.099 & 0 & 0.099 & 0 & 0.099 \\
\hline 2000 & 5 & 0.099 & 2 & 0.099 & 0 & 0.099 & 0 & 0.099 \\
\hline 3000 & 11 & 0.099 & 2 & 0.099 & 2 & 0.099 & 1 & 0.099 \\
\hline 4000 & 24 & 0.099 & 2 & 0.099 & 3 & 0.099 & 2 & 0.099 \\
\hline 5000 & 36 & 0.099 & 2 & 0.099 & 5 & 0.099 & 4 & 0.099 \\
\hline
\end{tabular}




\begin{tabular}{|c|c|c|c|c|c|c|c|c|}
\hline 6000 & 48 & 0.099 & 2 & 0.099 & 5 & 0.099 & 3 & 0.099 \\
\hline 8000 & 84 & 0.099 & 3 & 0.102 & 6 & 0.102 & 3 & 0.099 \\
\hline 10000 & 117 & 0.102 & 9 & 0.102 & 8 & 0.102 & 7 & 0.102 \\
\hline 12000 & 155 & 0.101 & 19 & 0.102 & 18 & 0.101 & 15 & 0.102 \\
\hline 14000 & 180 & 0.101 & 30 & 0.101 & 28 & 0.1 & 25 & 0.101 \\
\hline 16000 & 216 & 0.102 & 42 & 0.101 & 40 & 0.101 & 38 & 0.101 \\
\hline 18000 & 242 & 0.106 & 54 & 0.1 & 53 & 0.1 & 53 & 0.103 \\
\hline 20000 & 280 & 0.105 & 76 & 0.102 & 78 & 0.101 & 74 & 0.106 \\
\hline 22000 & 320 & 0.106 & 101 & 0.103 & 105 & 0.101 & 98 & 0.107 \\
\hline 24000 & 350 & 0.105 & 120 & 0.103 & 122 & 0.103 & 111 & 0.106 \\
\hline 26000 & 380 & 0.105 & 143 & 0.102 & 148 & 0.102 & 130 & 0.107 \\
\hline 28000 & 415 & 0.105 & 158 & 0.102 & 160 & 0.103 & 152 & 0.107 \\
\hline 30000 & 432 & 0.106 & 178 & 0.102 & 179 & 0.104 & 172 & 0.11 \\
\hline 32000 & 462 & 0.106 & 191 & 0.102 & 195 & 0.102 & 187 & 0.112 \\
\hline 34000 & 480 & 0.102 & 210 & 0.102 & 208 & 0.102 & 205 & 0.107 \\
\hline 36000 & 513 & 0.105 & 217 & 0.102 & 220 & 0.102 & 213 & 0.107 \\
\hline 38000 & 564 & 0.106 & 237 & 0.103 & 240 & 0.103 & 231 & 0.108 \\
\hline 40000 & 594 & 0.106 & 261 & 0.103 & 265 & 0.104 & 256 & 0.108 \\
\hline 42000 & 632 & 0.105 & 279 & 0.103 & 280 & 0.104 & 275 & 0.109 \\
\hline 43200 & 653 & 0.105 & 286 & 0.103 & 290 & 0.105 & 283 & 0.109 \\
\hline
\end{tabular}

From Figure 4, duplication avoidance outperformed other strategies as it had higher delivery probability almost throughout the simulation time. In general, congestion control using buffer size advertisement and the data centric methods reduced the number of packet loss but did not improve the delivery probability of the messages significantly. This is because there is no end-to-end connection in an opportunistic network. Hence, nodes are using any slight opportunity available to send messages. These forms of congestion control prevent nodes from accepting all messages, (due to buffer size or based on interest), as a result reducing forwarding opportunity (forwarding probability) and consequently the delivery probability. The use of acknowledgement did not improve the delivery probability because of the absence of end-to-end connectivity in an opportunistic network. The acknowledgement is either not received at all or not received in reasonable time. Hence, nodes still keep the messages in their buffer long after it has reached its destination. This is not an effective way of preventing congestion in an opportunistic network. The duplication avoidance reduced the number of packet loss as well as improved the delivery probability. This is because this method did not block any chance of forwarding messages except those messages that already existed in the node. Hence it did not decrease forwarding probability and it saved buffer space by preventing duplication. This resulted in an improved delivery probability.

Duplication avoidance improved the integrated routing better because it reduced the packet loss and improved the delivery probability. Duplication avoidance reduced the packet loss to 283 (58\% improvement) and improved the delivery probability to $0.109(4 \%)$ at the end of the simulation time. The use of acknowledgement, buffer size advertisement, data centric method reduced the packet loss to $653(2.5 \%$ improvement), 286 (57\% improvement) and 290 (57\% improvement) respectively but did not improve on the delivery probability significantly. Their delivery probabilities are $0.105,0.103$ and 0.105 respectively at the end of the simulation time. The delivery probability and packet loss of the integrated routing protocol without congestion control are .1048 and 670 respectively.

\section{CONCLUSION}

This work presents the application of congestion control on the integrated routing protocol. Four pre-emptive congestion control strategies (acknowledgement, buffer size advertisement, data centric method and the avoidance of duplication) were used. These results showed that, duplication avoidance improved the performance of the integrated routing protocol better (in terms of delivery probability and packet loss) than other congestion control strategies.

Security measures can be considered in the integrated routing of the opportunistic networks since malicious devices are likely to join the opportunistic network because of lack of initial authentication. All the congestion control strategies can be integrated as a hybrid congestion control strategy and used to further improve the performance of the integrated routing protocol. Extensive experimental setup (like the vehicular mobility model) can be used to evaluate the performance of the integrated routing protocol.

\section{REFERENCES}

[1] Lilien, L., Kamal, Z. H., Bhuse, V., and Gupta, A. 2006. Opportunistic networks: The concept and research challenges in privacy and security. Proc. of the WSPWN, 134-147. 
[2] Kaur, E. U., and Kaur, E. H. 2009. Routing techniques for opportunistic networks and Security Issues. National Conference on Computing, communication and control.

[3] Yogi, M. K., and Chinthala, V. 2014. A Study of Opportunistic Networks for Efficient Ubiquitous Computing. International Journal of Advanced Research in Computing and Communication Engineering .3(1), 5187-5191

[4] Asgari, C., Zareie, A., and Torkashvand, R. R. 2013. Intelligent Routing for Opportunistic Networks Based on Distributed Learning Automata. Journal of Basic and Applied Science Research, 3(7) 117-126.

[5] Verma, A., and Srivastava, D. 2012. Integrated routing protocol for opportunistic networks. arXiv preprint arXiv $: 1204.1658$

[6] Lindgren, A., Doria, A., and Schelen, O. 2003. Epidemic Routing for Partially Connected Adhoc Networks. ACM Mobile Computing and Communications Review, 7, 1920.

[7] Vahdat, A., and Becker, D. 2000. Epidemic Routing for Partially Connected Ad Hoc Networks. Technical Report CS-2000-06, Computer Science Department. Duke University.

[8] Silva, Aloizio P, Burleigh, Scott, Hirata, Celso M, and Obraczka, Katia. 2015. A survey on congestion control for delay and disruption tolerant networks. Ad Hoc Networks, Elsevier 25, 480-494.

[9] Pan, D., Ruan, Z., Zhou, N., Liu, X and Song, Z. 2013. A Comprehensive-integrated buffer management strategy for opportunistic Network. EURASIP Journal on Wireless Communication and Networking. 2013: 103

[10] Moreira, W., Mendes, P., and Sargento, S. 2011. Assessment model for opportunistic routing. Paper presented at the Communications (LATINCOM), 2011 IEEE Latin-American Conference on, 1-6.

[11] IP, Y .K.., Lau, W .C. and Yue, O. C. 2007. "Forwarding and replication strategies for DTN with resource constraints," In Proceedings of IEEE Vehicular Technology Conference, vol. 1, pp. 1260-1264.
[12] Keranen, A., and Ott, J. 2009. The ONE Simulator for DTN Protocol Evaluation. Special Report, Helsinki University of Technology, Networking Laboratory.

[13] Spyropoulos, T., Psounis, K. and Raghavendra, C. S. 2005 Spray and wait: an efficient routing in intermittently connected mobile networks. Proceeding of ACM SIGCOMM Workshop on Delay Tolerant Networking (WDTN), Philadelphia, USA, pp. 252-259.

[14] Santos, R.M., Orozco, J. and Ochoa, S. 2011. A real-time analysis approach in opportunistic networks. ACM SIGBED Rev. 8(3).

[15] Journi, K. and Jorg, O. 2008. Time scales and delaytolerant routing protocols. Proceedings of CHANTS'08, San Francisco, California, USA, pp. 13-19.

[16] Nguyen, H. A., Giordano, S. and Puiatti, A. 2007. Probabilistic routing protocol for intermittently connected mobile Ad hoc networks. Proceedings of IEEE International Symposium on a World of Wireless, Mobile and Multimedia Networks, Espoo, Finland, pp.1-

[17] Bjurefors, F. 2014. Opportunistic networking: Congestion, Transfer Ordering and Resilience. http://uu.divaportal.org/smash/get/diva2:713179/FULLTEXT01.pdf

[18] Oliveira, A. B., del, D. A, V., da Hora, D. N., and Macedo, D. F. 2014. Evaluating contacts in opportunistic networks over more realistic simulation models. Journal of Applied Computing Research, 3(1), 54-63.

[19] Huang, C.-M., Lan, K.-c., and Tsai, C.-Z. 2008. A survey of opportunistic networks. Paper presented at the Advanced Information Networking and ApplicationsWorkshops, 2008. AINAW 2008. 22nd International Conference on.

[20] Lindgren, A., Doria, A., and Schelen, O. 2003 Probabilistic Routing in intermittently connected Network, ACM Mobile Computing and Communications Review, Vol.7, pp.19-20. 